\title{
HEXASPIRON NIGERIENSIS DOLLFUS et GOLVAN, 1956 (EOACANTHOCEPHALA) EST UN GYRACANTHOCEPHALA
}

\author{
Y. J. GOLVAN*
}

SUMMARY. Nearly thirty specimens of Hexaspiron nigeriensis from the intestine of the fish Heterosynodontis membranaceus captured in the Niger river, gave the opportunity of a redescription of this acanthocephalan species. The presence of 7 crowns of minute spines on the fore part of the trunk obliged to transfert $H$. nigeriensis from Neoechinorhynchidae family into Quadrigyridae family.

Key-words: Acanthocephala. Hexaspiron nigeriensis. Africa. Parasites of fishes.

En 1956, mon maître Robert Ph. Dollfus et moi-même avons décrit un nouveau genre d'Acanthocéphale Hexaspiron avec, pour espèce type, Hexaspiron nigeriensis, parasite de l'intestin d'un Silure Mochocidae africain, Hemisynodontis membranaceus (Et. Geoffroy Saint-Hilaire, 1809). Nous ne disposions que de 5 exemplaires de cette espèce en assez mauvais état de préparation et de fixation. Louis Euzet vient de nous rapporter de Bamako (Niger) une trentaine d'exemplaires de cette espèce provenant du même hôte. Ce matériel nouveau est bien conservé et en l'étudiant, il s'avère que tous les individus présentent des couronnes de très petites épines sur la partie antérieure du tronc, détail qui m’avait échappé lorsque j'avais examiné les spécimens qui ont servi à la description princeps.

\section{Complément à la description originale}

Quelques spécimens seulement ont un proboscis parfaitement évaginé, chez d'autres le rostre est rétracté au fond du réceptacle, chez quelques-uns enfin il est invaginé en doigt de gant (fig. 4). Lorsque le proboscis est rétracté, la partie antérieure du tronc forme une sorte de tube qui est transversalement orné de 7 bandes noirâtres. A un grossissement plus fort, ces bandes apparaissent formées de couronnes de très petites épines, dont la pointe est alors dirigée vers l'avant, et qui sont au nombre de 12 à 16 . Ces épines s'insèrent sur des bases formées de

* Université Pierre-et-Marie-Curie, Faculté de Médecine Paris-Saint-Antoine, Laboratoire de Parasitologie, 27, rue Chaligny, F 75571 Paris Cedex 12.

Accepté le 28 mars 1988. 
granules très réfringents dont certains, particulièrement gros, dessinent une sorte de V. Lorsque le rostre est évaginé, ces couronnes couvrent la partie toute antérieure du tronc, juste en arrière de l'insertion du cou. Elles sont très petites, mesurant à peine $0,01 \mathrm{~mm}$ pour les plus grosses (fig. 3).

Les lemnisques sont à peine plus longs que le réceptacle, larges et plats.

Les individus mâles sont beaucoup plus petits que les femelles. Le testicule postérieur chevauche le pôle postérieur du testicule antérieur et il est à peine plus petit (alors que dans la description originale nous avions noté qu'il était beaucoup plus petit, caractère qui est donc sans valeur spécifique). Quelques spécimens ont la bourse caudale évaginée. Le pénis est alors visible sous forme d'un petit organe conique centrant la bourse (fig. 1).

La plus grande femelle dont je disposais mesurait entre 7,5 et $8 \mathrm{~cm}$ de long, les œufs étaient en voie de maturation et seuls quelques-uns d'entre eux étaient mûrs. Ils présentent alors une coque externe extrêmement mince (fig. \%) souvent plissée et facilement caduque.

La vulve est nettement sub-terminale (fig. 2 et 5). J'ai compté six cellules sélectrices à la base de la cloche utérine. Il y a deux gros sphincters à la base du tube utérin, plus un manchon musculaire vaginal bien développé.

Il m'a été impossible de voir les noyaux géants sous-cuticulaires, non plus que ceux de la glande cémentaire.

Les canaux lacunaires dorsal et ventral, très gros, sont, au moins dans la partie postérieure du tronc, anastomosés en réseau alors qu'ils prennent plutôt une disposition transversale pseudo-métamérique dans la partie antérieure.

\section{Position systématique}

La présence d'épines cuticulaires sur la partie antérieure du tronc oblige à classer le genre Hexaspiron dans l'Ordre des Gyracanthocephala Van Cleave, 1936 et non dans celui des Neoechinorhynchida Southwell et MacFie, 1925 comme nous l'avions proposé en 1956.

Il appartient donc à la Famille des Quadrigyridae Van Cleave, 1920 qui est la seule reconnue dans cet ordre. La nouvelle diagnose du genre Hexaspiron Dollfus et Golvan, 1956 doit s'énoncer ainsi :

Gyracanthocephala de taille moyenne (entre 7 et $10 \mathrm{~cm}$ ). Dimorphisme sexuel important, les femelles étant beaucoup plus grandes que les mâles. Proboscis très petit, sphéroïdal, armé de 6 spires de 4 crochets dont la taille décroît de l'apex à la base du rostre et insérés sur des racines de forme simple. Proboscis souvent rétracté dans le réceptacle ou même invaginé en doigt de gant. Grosse papille sensorielle apicale. Cou tronc-conique. Réceptacle à paroi simple formée de fibres obliques. Ganglion cérébroïde volumineux, placé au fond du réceptacle. Lemnisci aplatis, à peine plus longs que le réceptacle. Partie antérieure du tronc invaginable et ornée de 7 couronnes complètes de 12 à 16 épines très petites insérées sur des 


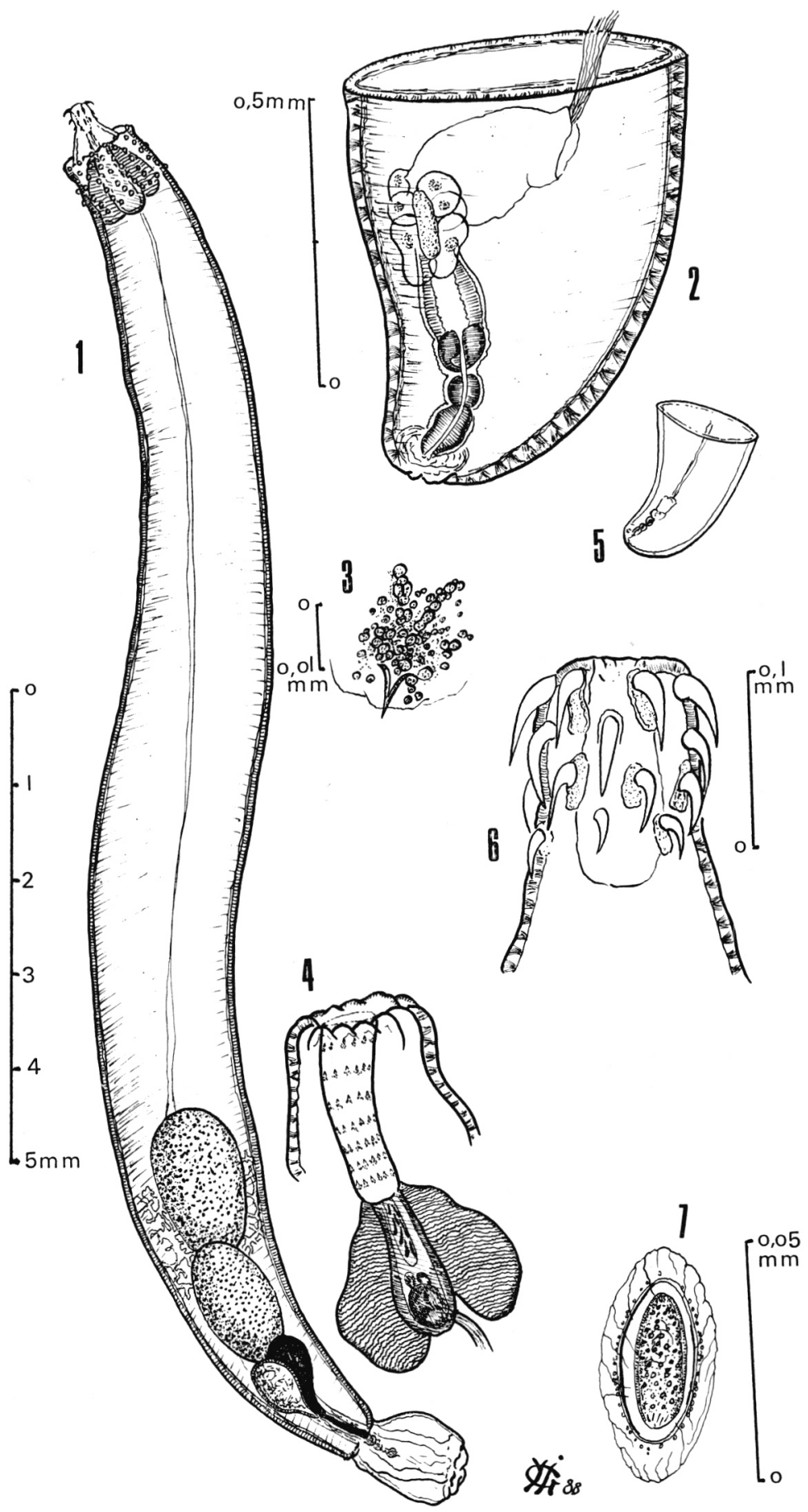

FIG. 1. - Individu mâle d'Hexaspiron nigeriensis, Dollfus et Golvan, 1956.

FIg. 2. - Appareil génital femelle d'H. nigeriensis.

Fig. 3. - Epine cuticulaire du tronc.

FIG. 4. - Partie antérieure du tronc et proboscis invaginé en doigt de gant.

FIg. 5. - Vulve en position sub-terminale.

FIG. 6. - Proboscis.

Fig. 7. - Embryophore mûr. 
bases granuleuses. Mâles à tronc fusiforme, femelles à corps sensiblement cylindrique, souvent enroulé en " ressort de montre " ou en " tire-bouchon ". Canaux principaux du système lacunaire dorsal et ventral de grande largeur, unis entre eux par des anastomoses sensiblement transversales vers l'avant et dessinant, vers l'arrière, un réseau à larges mailles. Noyaux sous-cuticulaires sans doute fragmentés. Appareil génital mâle occupant à peine le $1 / 4$ postérieur du tronc. Testicules ovoïdes, placés l'un derrière l'autre et en contact, l'antérieur un peu plus gros que le postérieur. Glande cémentaire unique syncytiale, pyriforme. Organe de Säfftigen placé un peu en arrière. Pénis tronc-conique. Appareil génital femelle très petit, n'occupant qu'une fraction infime de la longueur du tronc. Deux gros sphincters entourant la partie terminale du tube utérin. Manchon musculaire important péri-vaginal. Vulve nettement sub-terminale. Embryophores ovoïdes à coque externe très mince et souvent plissée. Parasites de l'intestin de poissons d'eau douce africains. Espèce type : Hexaspiron nigeriensis, Dollfus et Golvan, 1956.

\section{BIBLIOGRAPHIE}

Dollfus R. Ph., Golvan Y. J. : Mission M. Blanc et F. d’Aubenton (1954). V. Acanthocéphales des Poissons du Niger. Bull. Instit. Franç. Afrique Noire, 1956, 18 (sér. A, no 4), 1086-1106. 